\title{
Rapid and non-destructive detection method for water status and water distribution of rice seeds with different vigor
}

\author{
Ping Song ${ }^{1,2}$, Ghiseok Kim ${ }^{3}$, Peng Song ${ }^{4}$, Tao Yang ${ }^{5,6 *}$, Xia Yue ${ }^{1,2}$, Ying Gu ${ }^{1,2}$ \\ (1. College of Information and Electrical Engineering, Shenyang Agricultural University, Shenyang 110866, China; \\ 2. Liaoning Engineering Research Centre for Information Technology in Agricultural, Shenyang Agricultural University, \\ Shenyang 110866, China; \\ 3. Department of Biosystems \& Biomaterials Science and Engineering, College of Agriculture \& Life Sciences, \\ Seoul National University, Seoul 08826, Korea; \\ 4. College of Plant Science \& Technology, Huazhong Agricultural University, Wuhan 430070, China; \\ 5. School of Information and Intelligence Engineering, University of Sanya, Sanya, Hainan 572022, China; \\ 6. Academician Workstation of Guoliang Chen, University of Sanya, Sanya, Hainan 572022, China)
}

\begin{abstract}
In this study, newly harvested and aged rice seeds were analyzed to determine their aging process, identify the difference between artificially and naturally aged seeds, and develop a rapid, accurate, and non-destructive detection method for water status and water distribution of rice seed with different vigor. To this end, an artificially accelerated aging test was conducted on the newly harvested rice seeds. Then, low-field nuclear magnetic resonance (LF-NMR) technology was applied to test the new (Shennong No. 9816, 2018), old (Shennong No. 9816, 2017), and artificially aged seeds (Shennong No. 9816, 2018). A standard germination test was conducted for three types of seeds. Finally, the differences of water status and distribution between rice seeds of different vigor were analyzed based on the standard germination test results and wave spectrometry information collected using LF-NMR. The results indicated that new seeds, old seeds, and the artificially accelerated aging rice seeds all exhibited two water phases, and the vigor of rice seeds after the artificial accelerated aging test was lower than that of new seeds. There were significant differences between the frequencies of bound water at the time of the peak and the time at the end of the peak for the three types of seeds. The two times showed an increasing trend for rice seeds with poor vigor, indicating that the ability of the water in the rice seeds having poor vigor to combine with other substances was weakened. There were significant differences between the distributions of free water peak end time for the three types of seeds. All the rice seeds with poor vigor exhibited a decreasing trend at this time, indicating that the freedom of free water inside the rice seed samples with poor vigor was weakened. The total water content of the artificially aged seeds and the aged seeds was higher than that of the new seeds, but the free water content increased from artificially aged seeds to new seeds to aged seeds. This indicates that LF-NMR technology is an effective detection method that can simply compare the differences in seed vitality with respect to water distribution as well as differentiate the seed internal water content of artificially aged and naturally aged seeds.
\end{abstract}

Keywords: low-field nuclear magnetic resonance, rice seed, water status detection, water distribution detection, seed vigor DOI: $10.25165 /$ j.ijabe.20211402.5780

Citation: Song P, Kim G, Song P, yang T, Yue X, Gu Y. Rapid and non-destructive detection method for water status and water distribution of rice seeds with different vigor. Int J Agric \& Biol Eng, 2021; 14(2): 231-238.

\section{Introduction}

Seed vigor is a comprehensive manifestation of seed characteristics that measure the activity and performance of a seedlot with acceptable germination rate in a wide range of environments $^{[1]}$. High vigor seeds can germinate and emerge

\section{Received date: 2020-03-17 Accepted date: 2021-01-04}

Biographies: Ping Song, PhD, Professor, research interest: precision agriculture Email: songping@syau.edu.cn; Ghiseok Kim, PhD, Associate Professor, research interest: hyperspectral technique, Email: ghiseok@snu.ac.kr; Peng Song, $\mathrm{PhD}$, Associate Professor, research interest: plant information nondestructive acquisition technology and equipment, Email: songp@ mail.hzau.edu.cn; Xia Yue, Master candidate, research interest: nuclear magnetic resonance, Email: 13220819559@163.com; Ying Gu, Master candidate, research interest: nuclear magnetic resonance, Email: 2858968579@qq.com.

*Corresponding author: Tao Yang, $\mathrm{PhD}$, Professor, research interest: computer technology in agriculture. School of Information and Intelligence Engineering, University of Sanya, Sanya, Hainan 572022, China. Tel: +86-13840296051, Email: 328748306@qq.com. rapidly and uniformly under extensive conditions, and the seedlings can grow vigorously with a strong resistance against adverse environmental conditions. In contrast, the germination and emergence speeds of low vigor seeds are low. Under adverse conditions, their emergence is not uniform, the seedlings are weak, and sometimes, there is no emergence at all ${ }^{[2,3]}$. Seed vigor is an important indicator of sowing quality and a critical component of planting value, and it is closely associated with planting production $^{[4]}$. With an increase in storage time, the rice seed aging, seed vigor, germination rate, and germination potential decrease, which reduces the seed value of rice.

Presently, there are multiple methods for seed vigor testing, which can be divided mainly into direct and indirect methods. Direct methods simulate adverse field conditions under a laboratory environment to measure the field emergence rate of a seedlot. These include low temperature germination test and brick gravel test. Indirect methods, which include conductivity measurement and tetrazolium tests, determine certain physiological and biochemical indicators that are related to seed vigor. Traditional 
seed vigor testing methods are capable of accurately and intuitively predicting the seed vigor, but they have disadvantages such as a large workload, poor repeatability, long cycle, significant environmental impact, and potential to damage the seeds. Therefore, new technologies have continuously been applied to improve seed vigor testing, such as near-infrared spectroscopy and infrared imaging. Obtaining a fast, accurate, and nondestructive method to determine seed vigor has become a current research hotspot ${ }^{[5]}$. Infrared spectroscopy provides the characteristic vibration frequency of functional groups or chemical bonds, and nuclear magnetic resonance spectroscopy provides the number of nuclei and the chemical environment. The former is more suitable for qualitative analysis in terms of application scope and information, while the latter is more accurate for quantitative analysis. As a novel nondestructive testing technology, low-field nuclear magnetic resonance (LF-NMR) and the associated imaging technology are widely used in the field of food agriculture, such as in the detection of oil and water content ${ }^{[6,7]}$; the investigation of the distribution, migration, and mobility of water and oil ${ }^{[8-11]}$; quality testing, processing, and storage of meat, aquatic, and agricultural products $^{[12-17]}$; and seed selection, breeding, and development ${ }^{[18,19]}$. However, its application to water status and the water distribution detection of rice seeds for testing different vigor is rarely reported.

Natural aging refers to the natural decline of seed vigor, and the process is very slow. In the laboratory, artificial accelerated aging test is to let the seeds grow or die in the aging test box by simulating the humidity, light, temperature and other parameters of the natural environment. Using new harvest and aged rice seed as the research object, this project firstly used the artificial accelerated aging test for the new harvest rice seeds. Then, LF-NMR technology was used for both naturally aging and artificially aging rice seeds. Finally, a standard germination test was conducted. The LF-NMR method can be used to perform wave spectrometry directly without sample pretreatment, and can help to perform a rapid wave spectrometry analysis for a single sample within 1 minute to directly obtain the chemical composition information of relevant substances, so as to achieve rapid, accurate, and non-destructive testing. This project attempts to explore a detection method for the water status and water distribution of rice seeds with different vigor, while reflecting the vigor of rice seeds with respect to water distribution and water content changes. The difference between the artificial and natural aging of rice seeds was compared to provide a reference for the storage of rice seeds and study and utilization of seed resources.

\section{Materials and methods}

\subsection{Test equipment}

The following equipment was used in this study: a magnetic resonance imaging analyzer NMI20-015V-I (Shanghai Niumai Electronic Technology Co., Ltd., Shanghai, China) with a magnetic field strength of $(0.5 \pm 0.08) \mathrm{T}$, radio frequency (RF) pulse frequency of $21 \mathrm{MHz}$, magnet temperature of $32^{\circ} \mathrm{C}$, and probe coil diameter of $15 \mathrm{~mm}$; an intelligent artificial climate incubator RTOP-268D (Zhejiang Tuopu Instrument Co., Ltd., Zhejiang, China) with a temperature control range of $0^{\circ} \mathrm{C}-50^{\circ} \mathrm{C}$, temperature fluctuation of $\pm 0.5^{\circ} \mathrm{C}$, temperature uniformity of $\pm 1{ }^{\circ} \mathrm{C}$, relative humidity control range of $50 \%-95 \%$, and relative humidity fluctuation of $\pm 5 \%$; a seed aging cabinet (Zhejiang Tuopu Instrument Co., Ltd., Zhejiang, China) with a temperature control range from room temperature plus $5^{\circ} \mathrm{C}$ to $65^{\circ} \mathrm{C}$ and timing range of 1-9999 m; and a Sartorius BAS124S-CW ten-thousandth electronic balance internally calibrated (Beijing Sartorius Instrument System Co., Ltd., Beijing, China) with a maximum weighing value of $120 \mathrm{~g}$, minimum weighing value of $0.01 \mathrm{~g}$, and accuracy of $0.0001 \mathrm{~g}$.

\subsection{Test materials}

All the test samples were obtained from the Rice Research Institute of Shenyang Agricultural University. The samples were harvested in October 2017 and October 2018 at the Liaozhong, Liaoning, China. The experiment was conducted in November 2018 in the Rice Experimental Station of Shenyang Agricultural University. The rice variety used was Shennong No. 9816, which is a Japonica rice. The seeds were stored at room temperature. A total of 1200 mature rice seeds, which were large, full, and free of disease spots on the surface, were selected (including 400 rice seeds harvested in 2017 and 800 rice seeds harvested in 2018) and divided into 12 groups with 100 seeds in each group. Then, according to the corresponding test plan, each group was marked with a unique number. Information of the specific grouping and test item is listed in Table 1

Table 1 Rice seed test sample grouping and test item

\begin{tabular}{cccccc}
\hline Type & Year & Group amount & Label & Seeds amount per group & Test item \\
\hline New seeds & 2018 & 4 & $\mathrm{~A}_{1}, \mathrm{~B}_{1}, \mathrm{C}_{1}, \mathrm{D}_{1}$ & 100 & LF-NMR test, standard germination test \\
Old seeds & 2017 & 4 & $\mathrm{~A}_{2}, \mathrm{~B}_{2}, \mathrm{C}_{2}, \mathrm{D}_{2}$ & 100 & LF-NMR test, standard germination test \\
Aged seeds & 2018 & 4 & $\mathrm{~A}_{3}, \mathrm{~B}_{3}, \mathrm{C}_{3}, \mathrm{D}_{3}$ & 100 & Accelerated aging test, LF-NMR test, standard germination test \\
\hline
\end{tabular}

\subsection{Test method}

\subsubsection{Artificial accelerated aging test}

According to the aging method of International Association for seed testing (ISTA), the aging group rice seeds were subjected to artificially accelerated aging. Four $12 \mathrm{~cm} \times 12 \mathrm{~cm} \times 6 \mathrm{~cm}$ plastic boxes with a cover were injected with deionized water up to $2 \mathrm{~cm}$ from the bottom. A stent was placed about $4 \mathrm{~cm}$ above the water surface, and a plastic plate with several circular meshes with a radius of $1.5 \mathrm{~mm}$ was placed on the top of the stent. Four groups of samples of the aged seeds were then evenly spread on the surfaces of the four plastic plates (to ensure that each seed absorbed water uniformly); subsequently, the cover was placed on the top to seal the samples in the boxes.

Next, the four test samples were simultaneously placed in a seed aging cabinet at a temperature of $45^{\circ} \mathrm{C}$ and relative humidity of $100 \%$ to age for $72 \mathrm{~h}$. Under the conditions of high temperature and high humidity, the content and distribution of water in rice seeds will change. Previous experiments have shown that under different aging humidity and temperature conditions, the phase state of bound water and free water of rice seeds will not change, but their distribution will be different. Each cabinet was separated by a gap of $3 \mathrm{~cm}$ to ensure a uniform temperature within the cabinet. The door was not opened during the aging treatment to accurately control the temperature in the cabinet. To prevent microbial contamination, the body, cover, and inner plastic plates of the aging cabinet were all immersed in a $15 \%$ sodium hypochlorite solution and then dried. After the aging treatment, the seeds were dried at $25^{\circ} \mathrm{C}$ for $3-4 \mathrm{~d}$ until the moisture content of the seeds was reduced to the original state. Then, LF-NMR spectroscopy and standard germination tests were conducted. 


\subsubsection{NMR spectroscopy analysis and inversion}

NMR spectroscopy was performed on 12 test samples, which included rice seeds harvested in 2018, rice seeds freshly harvested in 2019 , and rice seeds that underwent the artificially accelerated aging test. Prior to the test, each group of 100 rice seeds was further divided into 5 subgroups of 20 seeds each (to obtain 20 parallel samples for each test condition), resulting in a total of 60 NMR spectroscopy test groups. Each parallel sample was weighed on an electronic balance with a sensitivity of $0.0001 \mathrm{~g}$ and its mass was recorded.

The main frequency and hard pulse width of the NMR instrument were determined using free induction decay (FID) pulse sequences in a standard oil sample and the NMR analysis application software. The free decay signal curves of the 60 test groups were collected using the carr-purcell-meiboom-gill (CPMG) pulse sequence in the NMR application software, with four repeated measurements for each sample. The spin echo signals generated from these four measurements were then applied to the NMR inversion fitting software to perform individual inversion, and the averaged results were used to obtain the transverse relaxation time and signal amplitude of the samples.

The parameters of the CPMG pulse sequence were set as follows: $S F 1$ (spectrometer frequency) was $21 \mathrm{MHz}, O 1$ (frequency offset 1$)$ was automatically corrected for each experiment, $P 1\left(90^{\circ}\right.$ pulse length) was $17.52 \mu \mathrm{s}, P 2\left(180^{\circ}\right.$ pulse length) was $35.52 \mu \mathrm{s}$, $T D$ (the signal sampling point numbers) was $74990, T W$ (repeated sampling waiting time) was $2000 \mathrm{~ms}, P R G$ (pre-amplifier gain) was 3, NECH (echo number) was $3000, T E$ (wait time of repeated sampling) was $0.25 \mathrm{~ms}, S W$ (receiver receives the signal frequency) was $100 \mathrm{kHz}, R F D$ (radio frequency delay) was $0.08 \mathrm{~ms}$, $R G 1$ (transmission gain) was $20 \mathrm{~dB}, D R G 1$ (digital gain) was $1, D R$ (data radius) was 1 , and $N S$ (repeated sampling frequency) was 64 .

\subsubsection{Standard germination test}

The standard germination test for rice seeds after NMR collection was conducted while referring to the germination conditions in the international rules for seed inspection. The rice seeds were disinfected in a $3 \%$ hypochlorous acid solution and then rinsed with distilled water thrice. Then, they were evenly spread in the germination cabinet and covered with germination paper. An appropriate amount of distilled water was subsequently injected to promote germination (the amount of distilled water added should be sufficient to soak the filter paper and the seeds; a pipettor was used to remove the extra distilled water to allow an equal amount of distilled water to be added to each test sample). Next, each group of samples was placed in a smart artificial climate incubator at a temperature of $27^{\circ} \mathrm{C}$ to ensure a constant temperature culture for $6 \mathrm{~d}$, with the incubator set to a segmented mode with $12 \mathrm{~h}$ illumination and $12 \mathrm{~h}$ darkness. The water content of the filter paper was observed every day, and if necessary, replenished. The number of germinated seeds and the seedling height were also recorded. After $6 \mathrm{~d}$, the seedling height was measured, and seeds with a seedling height exceeding $4 \mathrm{~cm}$ were identified as high vigor seeds. The germination index $(G I)$, vigor index $(V I)$, and high vigor seed ratio $(H V R)$ were calculated using the formulas listed below.

$$
G I=\sum_{t=1}^{t=N} \frac{G t}{D t}
$$

where, $N$ is the number of days, $D t$ is the number of germination days and $G t$ is the corresponding number of germinated seeds per day.

$$
V I=G I \times S
$$

where, $S$ is the normal seedling height at a certain period, $\mathrm{cm}$.

$$
H V R=N_{1} / N_{2} \times 100 \%
$$

where, $N_{1}$ is the number of rice seeds with a seedling height greater than $4 \mathrm{~cm}$ after $6 \mathrm{~d}$ and $N_{2}$ is the number of seed samples.

\subsection{Data processing}

All data acquired in the LF-NMR spectroscopy and standard germination tests was processed using the SPSS 20.0 software. The data was expressed as the mean \pm standard deviation. To eliminate the influence of the initial quality of rice on the test results, the signal amplitudes of all the test samples were normalized by mass in groups of $0.5 \mathrm{~g}$. Data with a high degree of discreteness was carefully handled during processing, as differences in the sample composition, NMR instruments, or measurement parameters could yield different results.

\section{Results and discussion}

\subsection{Internal water phase division of new, old, and aged seed}

Figure 1 shows the $T_{2}$ relaxation spectrum of the samples including the relaxation time and the corresponding relaxation signal component, obtained by fitting and inverting the $T_{2}$ decay curve acquired by the CPGM pulse sequence into the relaxation model using an iterative optimization method. The abscissa is 100 transverse relaxation time components $T_{2}$ logarithmically distributed from $10^{-2} \mathrm{~ms}$ to $10^{4} \mathrm{~ms}$, and the ordinate is the signal component $A_{2 \mathrm{i}}$ corresponding to each relaxation time (to facilitate quantitative analysis, all signal components were normalized by mass). According to the NMR principle, this signal component is proportional to its component content. The integral amplitude $A$ is the sum of all components, which is the semaphore of the sample $\mathrm{e}^{[20-26]}$.

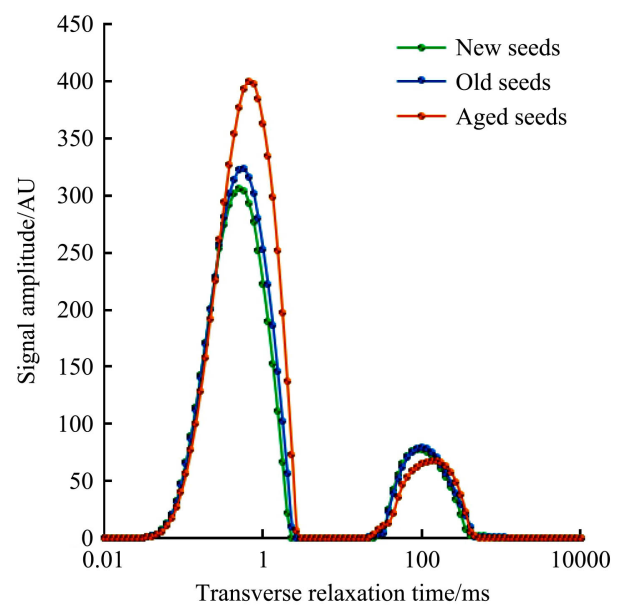

Note: The new seeds were Shennong No. 9816 rice seeds harvested in 2018, the old seeds were Shennong No. 9816 rice seeds harvested in 2017 , and the aged seeds were Shennong No. 9816 rice seeds harvested in 2018 and subjected to an artificially accelerated aging test.

Figure 1 Inversion spectrum of transverse relaxation time $T_{2}$ of rice seeds with different vigor levels

Spin-lattice relaxation (longitudinal relaxation time, $T_{1}$ ) and spin-spin relaxation (transverse relaxation time, $T_{2}$ ) are two primary LF-NMR parameters that characterize proton relaxation, of which $T_{1}$ measures the recovery curve, while $T_{2}$ measures the decay curve. Because of its high sensitivity and short acquisition, $T_{2}$ provides significantly more information about the relaxation time ( $T_{2}$ can measure 1024 data points, whereas $T_{1}$ can only measure 25 points), and is consequently becoming more and more popular. The $T_{2}$ relaxation time reflects the chemical environment of the 
hydrogen protons in the sample. It is related to the binding force and the degree of freedom of the hydrogen protons, whereas the degree of hydrogen proton binding is closely associated with the internal structure of the sample ${ }^{[27-32]}$. The greater the binding of hydrogen protons, i.e., the smaller the degree of freedom of hydrogen protons, the shorter the $T_{2}$ relaxation time, which corresponds to a location more towards the left in the $T_{2}$ inversion spectrum; however, for a longer $T_{2}$ relaxation time, the location is more toward the right ${ }^{[33-41]}$.

From the $T_{2}$ distribution curve of the rice seed samples in Figure 1, it can be seen that there are consistently two peaks in the $T_{2}$ inversion spectrum no matter the seed is new, old, or has undergone an artificial accelerated aging test. The water phase model of the relaxation spectrum was then analyzed according to the rice seed sample, and it was concluded that the relationship between the peak and the water phase can be described as follows: the first peak, $T_{21}$, whose relaxation time is less than $10 \mathrm{~ms}$, corresponds to the bound water content, and its signal amplitude is $A_{21}$. This water is absorbed by hydrophilic colloids such as starch and protein in the seeds owing to the gravitational force between molecules; however, it is difficult to evaporate from the seeds and hence, less mobile. The second peak, $T_{22}$, whose relaxation time is greater than $10 \mathrm{~ms}$, corresponds to the free water content, and its signal amplitude is $A_{22}$. This water is relatively loosely present in the seeds owing to capillary gravitation and can move freely. It exhibits the properties of ordinary water, exists in the intercellular space, and can be evaporated easily. The semaphore $A$ of the sample here is $A_{21}+A_{22}$. By analyzing Figure 1, it is found that the bound water contributes more significantly to the overall signal than the free water.

3.2 Transverse relaxation time differences for bound water content in new, old, and aged seeds

A total of 60 samples, including new seeds, old seeds, and seeds that underwent the accelerated aging test, were tested. The $T_{2}$ decay curves were measured separately, and the $T_{2}$ distribution curves were obtained by iterating the inversion algorithm 10000 times. The basic statistical information of the $T_{2}$ distribution relaxation time is listed in Table 2 .

Table 2 Transverse relaxation time for water phase division

\begin{tabular}{|c|c|c|c|c|}
\hline Group & Number & $\begin{array}{l}\text { Onset time of } \\
\text { peak } / \mathrm{ms}\end{array}$ & Peak time/ms & $\begin{array}{c}\text { End time of } \\
\text { peak } / \mathrm{ms}\end{array}$ \\
\hline \multirow{3}{*}{$T_{21}$} & New seeds & $0.01 \pm 0$ & $0.5174 \pm 0.0328$ & $2.31 \pm 0$ \\
\hline & Old seeds & $0.01 \pm 0$ & $0.5295 \pm 0.0368$ & $2.3328 \pm 0.1113$ \\
\hline & Aged seeds & $0.01 \pm 0$ & $0.6181 \pm 0.0433$ & $2.7796 \pm 0.2204$ \\
\hline \multirow{3}{*}{$T_{22}$} & New seeds & $21.6204 \pm 5.2942$ & $96.9172 \pm 12.9939$ & $615.1375 \pm 436.6305$ \\
\hline & Old seeds & $21.5652 \pm 4.8866$ & $97.6248 \pm 12.0601$ & $572.1727 \pm 324.4439$ \\
\hline & Aged seeds & $23.9896 \pm 4.9205$ & $105.4875 \pm 12.9402$ & $420.6590 \pm 59.5991$ \\
\hline
\end{tabular}

Note: The first peak: $T_{21}$, The second peak: $T_{22}$. All the statistical data is measurement data, and is expressed as mean \pm standard deviation.

An analysis of Table 2 reveals that, compared with new seeds, the peak time and the end time of $T_{21}$ peak of old seeds and artificial accelerated aging seeds exhibit an increasing trend, whereas the end time of $T_{22}$ peak exhibits a decreasing trend. This indicates that, compared with the new seeds, the binding ability of the internal combined water and other substances is weakened, and the degree of freedom of free water is weakened. Herein, all the data are analyzed further in terms of frequency, in an attempt to reveal its changing trend more clearly.

Figure 2 shows the differences in the transverse relaxation time of the internal bound water content (transverse relaxation time $T_{21}$, signal amplitude $A_{21}$ ) of new, old, and aged seeds. In all three figures, the ordinate represents the three types of rice seed samples and the abscissa denotes the number of seeds of the different time. The stacked column chart demonstrates the proportion of the number of seeds of each time.

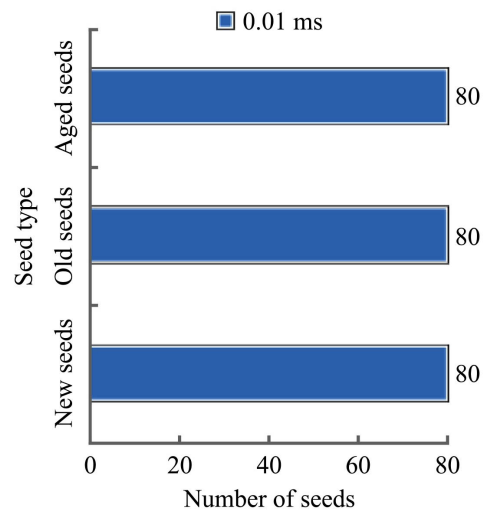

a. Onset time of peak

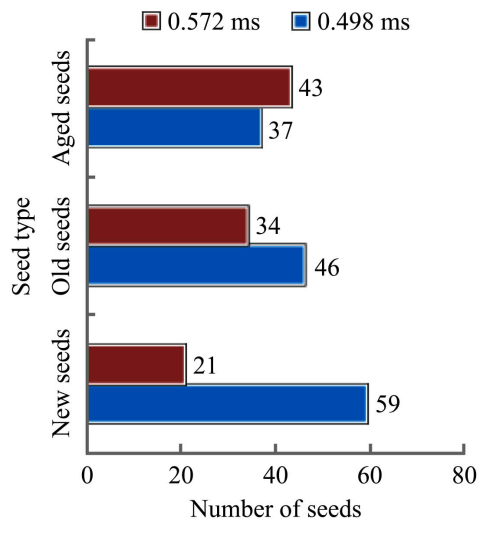

b. Peak time

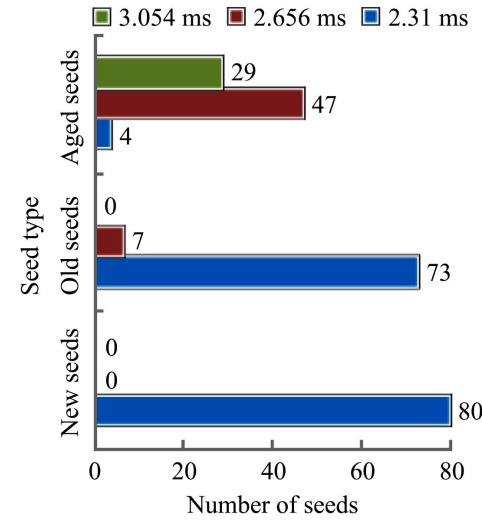

c. End time of peak

Note: The new seeds were Shennong No. 9816 rice seeds harvested in 2018, the old seeds were Shennong No. 9816 rice seeds harvested in 2017 , and the aged seeds were Shennong No. 9816 rice seeds harvested in 2018 and subjected to an artificially accelerated aging test. Figure a-c respectively show the number of seeds of peak start time, peak point time, and peak end time for different types of samples.

Figure 2 Transverse relaxation time differences for bound water content in new, old, and aged seeds

Figure 2a reveals that the internal bound water in the new, old, and aged seeds has the same peak onset time of 0.01 , which suggests that this parameter cannot be used to differentiate the vigor levels of the rice seed samples.

Figure $2 b$ indicates different proportions of the frequencies of the peak times for the different types of seeds. For the new seeds, the $T_{21}$ peak times of 0.498 and 0.572 correspond to proportions of $73.75 \%$ and $26.25 \%$, respectively; for the old seeds, they are $57.5 \%$ and $42.5 \%$, respectively; and for the aged seeds, they are $46.25 \%$ and $53.75 \%$, respectively. The average locations of the peak times are all towards the right. Compared with the new seeds, the time position of the peak points of the other two rice seeds exhibited a rightward-shifting trend.

Figure 2c depicts a more significant difference in the proportions of the frequencies of the end times of the peak for the different types of seeds. For the new seeds, the end times of the $T_{21}$ peak of $2.31 \mathrm{~ms}, 2.656 \mathrm{~ms}$, and $3.054 \mathrm{~ms}$ correspond to proportions of $100 \%, 0 \%$, and $0 \%$, respectively; for the old seeds, they are $91.25 \%, 8.75 \%$, and $0 \%$, respectively; and for the aged seeds, they are $5 \%, 58.75 \%$, and $36.25 \%$, respectively. Compared 
with the new seeds, the mean position of the peak end times of the other two types of rice seeds shifted more significantly to the right.

The transverse relaxation time reflects the binding ability of the internal water of the sample with other substances in the sample. Figure $2 \mathrm{~b}$ and Figure $2 \mathrm{c}$ indicate that this binding ability is weakened once the rice seeds are aged.

3.3 Transverse relaxation time differences for free water content in new, old, and aged seeds

Figure 3 shows the differences in the transverse relaxation time

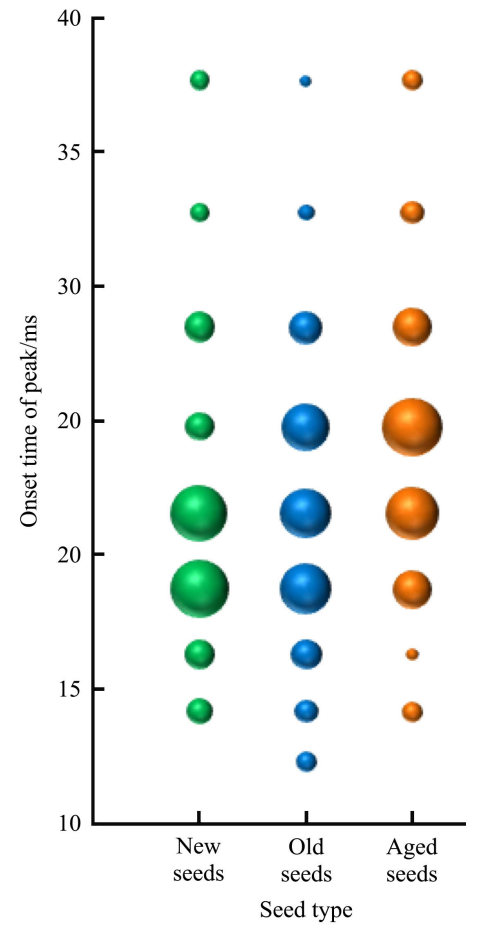

a. onset time of peak

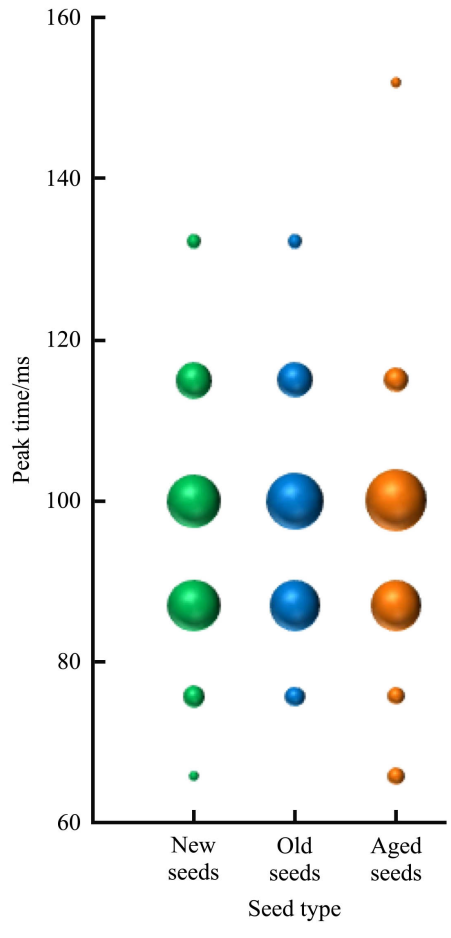

b. peak time of the internal free water content (transverse relaxation time $T_{22}$, signal amplitude $A_{22}$ ) of the new, old, and aged seeds. Figure $3 \mathrm{a}-\mathrm{c}$ respectively indicate the difference in the distribution of the peak onset time, peak time, and peak end time of the internal free water for the three types of rice seeds. Three colors are used in the figures to distinguish seeds with different vigor levels. The abscissa denotes the three types of rice seed samples and the ordinate indicates the time in milliseconds. The size of the bubble indicates the frequency of each time.

Note: The new seeds were Shennong No. 9816 rice seeds harvested in 2018, the old seeds were Shennong No. 9816 rice seeds harvested in 2017 , and the aged seeds were Shennong No. 9816 rice seeds harvested in 2018 and subjected to an artificially accelerated aging test. The size of the bubble indicates the frequency of each time.

Figure 3 Transverse relaxation time differences for free water content in new, old, and aged seeds

Figures $3 \mathrm{a}$ and $3 \mathrm{~b}$ reveal that there are no substantial differences in the distribution of the onset time and the peak time of the $T_{22}$ peak of the internal free water in the new, old, and aged seeds, suggesting that the end time of the $T_{22}$ peak reflect the seed vigor. However, the difference in the end-time distribution of the $T_{22}$ peak is extremely significant for the three seeds, as shown in Figure 3c. The figure reveals that the new seeds have the longest end time of the $T_{22}$ peak, followed by the old seeds and the aged seeds. This suggests that the free water in the new seeds has the highest degree of freedom, followed by that in the old seeds, with the free water in the aged seeds having the lowest degree of freedom.

\subsection{Effect of differences in seed vigor on signal amplitude in NMR inversion spectrum}

The existing state of the seed's water content is closely related to the life activities of the seed. When there is only bound water in the seed, the hydrolase in the seed is inactive, and the metabolism of the seed is extremely low. However, when the proportion of free water in the seed increases, the hydrolase goes into an activated state, causing the seed respiration intensity to rise and the seed vigor to decrease rapidly. Therefore, the internal water state and its distribution have an important impact on the seed vigor. The information on the signal amplitude and peak ratios of the three types of rice seeds is listed in Table 3. The peak ratio is the percentage of $A_{21}$ and $A_{22}$ in $A$.

According to the principle of LF-NMR, the amplitude of each peak reflects the water content of the different types and total water, and the radio of each peak reflects the proportion of the different types of water in total water. As presented in Table 3, compared with the new seeds, the combined water content in the seeds of the other two types of rice showed an increasing trend, the internal free water content changed irregularly, and the total water content exhibited an increasing trend. The proportion of combined water in the seeds of the other two types of rice increased, whereas the proportion of free water in the seeds decreased. To illustrate the differences among the three more intuitively, the combined water, free water, and total water moisture content were used to draw a three-variable bubble diagram, as shown in Figure 4.

Table 3 Signal amplitude and peak ratio for moisture phase division

\begin{tabular}{cccccc}
\hline Type & Signal amplitude of $A_{21} / \mathrm{AU}$ & Peak radio of $A_{21} / \%$ & Signal amplitude of $A_{22} / \mathrm{AU}$ & Peak radio of $A_{22} / \%$ & Signal amplitude of $A_{2} / \mathrm{AU}$ \\
\hline New seeds & $4567.417 \pm 109.116$ & $82.698 \pm 0.534$ & $942.250 \pm 44.751$ & $17.056 \pm 0.568$ & $5228.922 \pm 170.119$ \\
Old seeds & $4855.106 \pm 135.235$ & $82.899 \pm 0.809$ & $987.158 \pm 49.190$ & $16.860 \pm 0.811$ & $6058.313 \pm 325.032$ \\
Aged seeds & $5996.725 \pm 455.928$ & $86.451 \pm 1.023$ & $915.832 \pm 30.425$ & $13.281 \pm 1.013$ & $6814.125 \pm 216.982$ \\
\hline
\end{tabular}




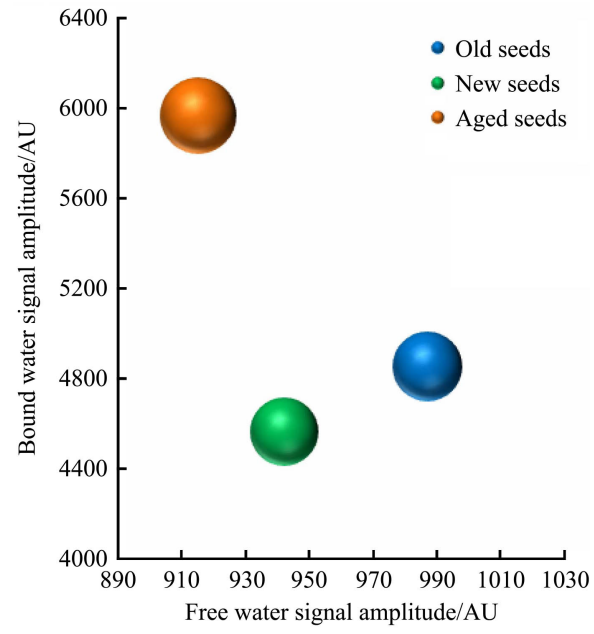

Note: The new seeds were Shennong No. 9816 rice seeds harvested in 2018, the old seeds were Shennong No. 9816 rice seeds harvested in 2017, and the aged seeds were Shennong No. 9816 rice seeds harvested in 2018 and subjected to an artificially accelerated aging test. The size of the bubbles indicates the overall signal amplitude of water in the samples.

Figure 4 Effect of differences in seed vigor of new, old, and aged seeds on signal amplitude in the NMR inversion spectrum

Figure 4 depicts the effects of the differences in seed vigor for the new, old, and aged seeds on the signal amplitude in the NMR inversion spectrum. The abscissa denotes the signal amplitude of free water, the ordinate represents the signal amplitude of bound water, and the size of the bubbles indicate the overall signal amplitude of water in the samples. A bubble chart was adopted as it best displays the relationship among the three variables of the three seed types.

Figure 4 suggests that after the seeds are aged, compared to the new seeds, the total amplitude of the $A$ peak in the other two types of rice seeds increases, indicating that the water content increases. The figure also indicates that the overall water content of the aged and old seeds is higher than that of the new seeds, which is consistent with the known conclusion that a very high internal water content results in reduced seed vigor, further validating the fact that LF-NMR technology is effective for testing rice seed vigor. If the internal water content of the seeds is too high, the respiratory metabolism of the seeds increases, which substantially accelerates the consumption of substances in seeds, thereby reducing seed vigor. In addition, seeds with a high-water content consume more oxygen owing to vigorous respiration, and as a result of hypoxia, they produce a large number of organic substances such as ethanol, which poisons the seed embryonic cells and causes them to lose vitality, resulting in reduced seed vigor.

Figure 4 also effectively shows the difference between artificially aged seeds and naturally aged seeds. Although the overall water contents of the two aged samples are both higher than that of the new seeds, the free water content of the artificially aged seeds is lower than that of the new seeds, while that of the naturally aged seeds is higher than that of the new seeds. This is because, during the artificially accelerated aging test, the rice seeds were placed in an environment at $45{ }^{\circ} \mathrm{C}$ and $100 \%$ relative humidity for continuous aging over $72 \mathrm{~h}$. The high temperature and humidity promoted cell metabolism, causing the cells to metabolize more vigorously, the concentration of organic carbohydrates such as fructose and glucose to continuously increase, and the water to convert to the bound state. Therefore, the bound water content significantly increased, while the free water content decreased.

Seeds are a colloidal substance with a porous capillary structure, whose surface and inner wall can absorb water vapor or gas molecules of other volatile substances. In addition, the water vapor or other volatile substances absorbed by the surface and the inner wall of the seeds can be released into the environment. This absorption and resolution process of the seed will reach a dynamic equilibrium after a certain period of time. Because old seeds are stored in air for a long time, the water in the air absorbed by the seeds exists in the free state. This free water, which reflects water solubility, metabolic activity, and membrane permeability, is highly mobile and can easily be dissipated in the rice seeds. When the proportion of the free water is sufficiently large, the primary viscosity of the substance reduces and the metabolism increases. At this stage, the concentration of carbohydrates that participate in the metabolism, such as glucose and fructose, will increase, causing the free water to transform into bound water, thereby reducing its concentration. Therefore, in old seeds, the distribution of free water and bound water vary. This study found that both increased by a small amount.

\subsection{Verification of differences in seed vigor of new, old, and} aged seeds using standard germination test

Figure 5 shows an image of the standard germination test used to determine the seed vigor of the samples. The image shown was taken on the 5th day of germination for a group of new seed test samples. The picture clearly indicates that the germination rate of this group is $100 \%$, and the heights of the seedlings vary.

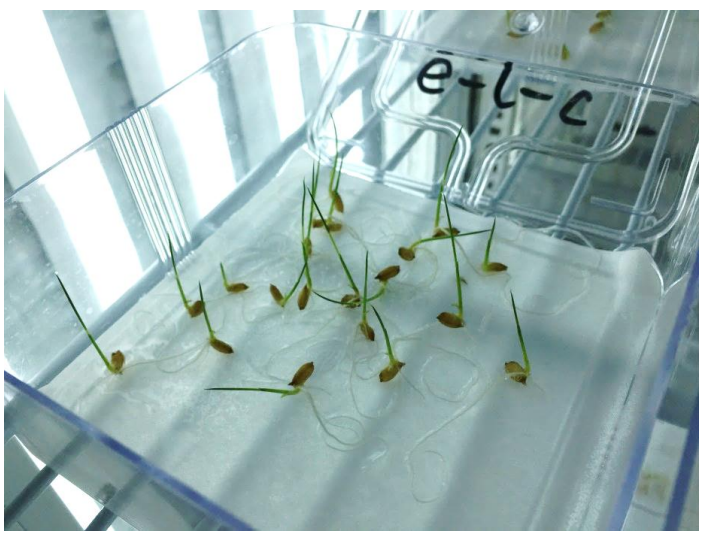

Figure 5 Rice seed standard germination test process

The number of germinations per day and the height of each seedling were recorded, and the $G I, V I$, and $H V R$ values were calculated using the equations given above. The values are listed in Table 4.

Table 4 Rice seed germination indices under standard germination test

\begin{tabular}{cccc}
\hline Type & $G I$ & $V I$ & HVR \\
\hline New seeds & 6.479 & 18.10 & $85.0 \%$ \\
Old seeds & 5.933 & 15.24 & $67.5 \%$ \\
Aged seeds & 6.229 & 16.73 & $75.0 \%$ \\
\hline
\end{tabular}

Note: The seeds were Shennong No. 9816 rice seeds harvested in 2018, the old seeds were Shennong No. 9816 rice seeds harvested in 2017, and the aged seeds were Shennong No. 9816 rice seeds harvested in 2018 and subjected to an artificially accelerated aging test.

The germination temperature was $27^{\circ} \mathrm{C}$ under $12 \mathrm{~h}$ illumination and $12 \mathrm{~h}$ darkness for $6 \mathrm{~d}$. GI is the germination index, $V I$ is the vigor index, and $H V R$ is the high vigor seed ratio. The data in Table 4 reveals that the GI, VI, and HVR of the new seeds are clearly higher than those of the naturally old seeds and artificially aged seeds. The process of the seed absorbing water and expanding, and the radicle breaking through the seed coat to reveal 
a white spot is called "sprouting" or "seed breaking", which is recognized as the start of germination during the tests and is thus recorded as "germination". However, the large number of test samples and variation in the observation angles introduced a high level of subjectivity. When the seed germ started to grow, the height of the seedling was measured and recorded daily, but the randomness of the growth direction and the seedling's curved growth feature made it more difficult to avoid human error in this part of the test. Therefore, the test results were considered valid only when the difference between the highest and the lowest values of four repeated measurements were within the maximum allowable range specified in the test process; the average was then used as the vigor level data for the batch of seeds.

The standard germination test verified the vigor level of the test samples and determined the standard adopted for the samples during the LF-NMR test. The standard germination test involved highlighted the convenience and speed of the LF-NMR nondestructive testing method, suggesting that this method of testing seed vigor can be used to obtain theoretical values and had good research prospects. The water status, distribution, and content of rice seeds with different vigor were observed. A multi-variety trial can be conducted to verify whether these changes can reflect the seed vigor level, which will be proved by further tests.

\section{Conclusions}

The internal bound water content (transverse relaxation time $T_{21}$, signal amplitude $A_{21}$ ) of the new, old, and artificially aged seeds demonstrated significantly different the peak time and the end time frequencies of the peak. For aged seeds, the binding ability of the internal water with other substances is weakened, increasing the transverse relaxation time.

The internal free water content (transverse relaxation time $T_{22}$, signal amplitude $A_{22}$ ) of the new, old, and artificially aged seeds demonstrated significantly different end times of the peak. The new seeds had the longest end time of the $T_{22}$ peak, followed by the old seeds and the aged seeds. This suggests that the free water in the new seeds has the highest degree of freedom.

The overall water content of the aged and old seeds is higher than that of the new seeds, which is consistent with the known fact that a very high internal water content leads to reduced seed vigor, further validating the fact that the LF-NMR technology is effective for testing rice seed vigor. In addition, the technology can effectively identify the differences of water content and water distribution of rice seeds between artificially aged and naturally aged seeds. Although the overall water content of the two aged samples with poor vigor is higher than that of the new seeds, the free water content of the naturally aged seeds is the highest followed by the new seeds and the artificially aged seeds. This suggests that LF-NMR technology can be used to easily identify seeds aged naturally and artificially based on their internal water distribution.

\section{Acknowledgements}

This project was supported by National Natural Science Foundation of China (Grant No. 31701318), National Natural Science Foundation of China Projects of International Cooperation and Exchanges (Grant No. 31811540396), and Basic Research Project of Education Department of Liaoning Province (Grant No. LSNJC201916).

\section{[References]}

[1] International Seed Inspection Association (ISTA). International rules for seed testing (2004 edition). Beijing: Technical Standards Press, 2004; 240p (in Chinese)

[2] Yan Q C. Seed. Beijing: China Agricultural Press, 2000; 559p. (in Chinese)

[3] $\mathrm{Hu}$ J. Seed storage and processing. Hangzhou: China Agricultural University press, 2001; 342 p. (in Chinese)

[4] Gao R Q, Zhang C Q. Crop seed science. Beijing: China Agricultural Press, 2015; 280p. (in Chinese)

[5] Makino Y, Ichimura M, Oshita S, Kawagoe Y, Yamanaka H. Estimation of oxygen uptake rate of tomato (Lycopersicon esculentum Mill.) fruits by artificial neural networks modelled using near-infrared spectral absorbance and fruit mass. Food Chemistry, 2010; 121(2): 533-539.

[6] Xu K, Lu J X, Gao Y L, Wu Y Q, Li X J. Determination of moisture content and moisture content profiles in wood during drying by low-field nuclear magnetic resonance. Drying Technology, 2017; 35(15): 1909-1908.

[7] Chen F L, Wei Y M, Zhang B. Characterization of water state and distribution in textured soybean protein using DSC and NMR. Journal of Food Engineering, 2010; 100(3): 522-526.

[8] Cao X, Zhang M, Mujumdar A S, Zhong Q, Wang Z. Measurement of water mobility and distribution in vacuum microwave-dried barley grass using Low-Field-NMR. Drying Technology, 2018; 36(15): 1892-1899.

[9] Pedersen H T, Munck L, Engelsen S B. Low-field ${ }^{1} \mathrm{H}$ nuclear magnetic resonance and chemometrics combined for simultaneous determination of water, oil, and protein contents in oilseeds. Journal of the American Oil Chemists Society, 2000; 77(10): 1069-1077.

[10] Li C, Peng A, He L, Ma S, Wu W, Yang H, et al. Emulsifying properties development of pork myofibrillar and sacroplasmic protein irradiated at different dose: A combined FT-IR spectroscopy and low-field NMR study. Food Chemistry, 2018; 252(30): 108-114.

[11] Mateus M L, Champion D, Liardon R, Voilley A. Characterization of water mobility in dry and wetted roasted coffee using low-field proton nuclear magnetic resonance. Journal of Food Engineering, 2007; 81(3): 572-579.

[12] Li M, Li B, Zhang W. Rapid and non-invasive detection and imaging of the hydrocolloid-injected prawns with low-field NMR and MRI. Food Chemistry, 2018; 242(1): 16-21.

[13] Khorshidi A S, Storsley J, Nkhata L, Joseph S. Advancing the science of wheat quality evaluation using nuclear magnetic resonance (NMR) and ultrasound-based techniques. Cereal Chemistry, 2018; 95(1): 347-364.

[14] Ribeiro R D O R, Mársico E T, Carneiro C D S, Monteiro M L G, Jesus E F $O$ D. Classification of Brazilian honeys by physical and chemical analytical methods and low field nuclear magnetic resonance (LF 1H NMR). LWT - Food Science and Technology, 2014; 55(1): 90-95.

[15] Wang S, Xiang W, Fan H, Xie J, Qian Y F. Study on the mobility of water and its correlation with the spoilage process of salmon (Salmo solar) stored at 0 and $4^{\circ} \mathrm{C}$ by low-field nuclear magnetic resonance (LF NMR 1H). Journal of Food Science \& Technology, 2017; 55(1): 1-10.

[16] Sun H X, Huang F, Ding Z J, Zhang C J, Zhang L. Low-field nuclear magnetic resonance analysis of the effects of heating temperature and time on braised beef. International Journal of Food Science \& Technology, 2017 52(5): 1193-1202.

[17] Bertram H C, Purslow P P, Andersen H J. Relationship between meat structure, water mobility, and distribution: A low-field nuclear magnetic resonance study. Journal of Agricultural and Food Chemistry, 2002; 50(4): 824-829.

[18] Khongsak S, Shafiur R M. Proton relaxation of waxy and non-waxy rice by low field nuclear magnetic resonance (LF-NMR) to their glassy and rubbery states. Journal of Cereal Science, 2018; 82(1): 94-98.

[19] Al-Habsi N A, Al-Hadhrami S, Al-Kasbi H, Rahman M S. Molecular mobility of fish flesh measured by low-field nuclear magnetic resonance (LF-NMR) relaxation: effects of freeze-thaw cycles. Fisheries Science, 2017; 83(5): 845-851.

[20] Zhang Q, Saleh A S M, Shen Q. Discrimination of edible vegetable oil adulteration with used frying oil by low field nuclear magnetic resonance. Food \& Bioprocess Technology, 2013; 6(9): 2562-2570.

[21] Wu J, Chen S. Investigation of the hydration of nonfouling material poly (ethylene glycol) by low-field nuclear magnetic resonance. Langmuir, 2012; 28(4): 2137-2144.

[22] Sanchez-Alonso I, Moreno P, Careche M. Low field nuclear magnetic resonance (LF-NMR) relaxometry in hake (Merluccius merluccius, L.) 
muscle after different freezing and storage conditions. Food Chemistry, 2014; 153(15): 250-257.

[23] Gudjónsdóttir M, Lauzon L H, Magnússon H, Sveinsdóttir K, Arason S, Martinsdóttir E, et al. Low field Nuclear Magnetic Resonance on the effect of salt and modified atmosphere packaging on cod (Gadus morhua) during superchilled storage. Food Research International, 2011; 44(1): 241-249.

[24] Hansen C L, Thybo A K, Bertram H C, Viereck N, Engelsen S B. Determination of dry matter content in potato tubers by low-field nuclear magnetic resonance (LF-NMR). Journal of Agricultural and Food Chemistry, 2010; 58(19): 10300-10304.

[25] Jiang C, Han J Z, Fan J L, Tian S Y. Rapid detection of adulterated milk by low field-nuclear magnetic resonance coupled with PCA method. Transactions of the CSAE, 2010; 26(9): 340-344. (in Chinese)

[26] Qi J, Gao F F, Li C B, Xu X L, Yang P Q. Changes of water holding capacity of mutton during freeze-thaw cycles by a low field nuclear magnetic resonance. Jiangsu Journal of Agricultural Sciences, 2010; 26(3): 617-622. (in Chinese)

[27] Sun J C, Zhang M, Gao Z, Xu B G, Bhesh B. Infusion of $\mathrm{CO}_{2}$ in a solid food: A novel method to enhance the low-frequency ultrasound effect on immersion freezing process. Innovative Food Science \& Emerging Technologies, 2016; 35(1): 194-203.

[28] Gudjónsdóttir M, Jónsson Á, Bergsson A B, Arason S, Rustad T. Shrimp processing assessed by low field nuclear magnetic resonance, near infrared spectroscopy, and physicochemical measurements-the effect of polyphosphate content and length of prebrining on shrimp muscle. Journal of Food Science, 2011; 76(4): 357-67.

[29] Xia T L, Liu D Y, Xu X L, Zhou G H, Shao J H. Application of low-field nuclear magnetic resonance in determining water contents and other related quality characteristics of meat and meat products: a review. Food science, 2011; 32(21): 253-256.

[30] Osán T M, Ollé J M, Carpinella M, Cerioni L M C, Pusiol D J, Appel M, et al. Fast measurements of average flow velocity by Low-Field 1H NMR. Journal of Magnetic Resonance, 2011; 209(2): 116-122.

[31] Carneiro C D S, Mársico E T, Ribeiro R D O R, Conte-Júnior C A, Mano S B, Augusto C J C, et al. Low-Field Nuclear Magnetic Resonance (LF NMR ${ }^{1} \mathrm{H}$ ) to assess the mobility of water during storage of salted fish (Sardinella brasiliensis). Journal of Food Engineering, 2016; 169(1): 321-325.

[32] Tan L, Wei C F, Tian H H, Zhou J Z, Wei H Z. Experimental study of unfrozen water content of frozen soils by low-field nuclear magnetic resonance. Yantu Lixue/Rock and Soil Mechanics, 2015; 36(6): 1566-1572. (in Chinese)

[33] Sanchez-Alonso I, Moreno P, Careche M. Low field nuclear magnetic resonance (LF-NMR) relaxometry in hake (Merluccius merluccius, L.) muscle after different freezing and storage conditions. Food Chemistry, 2016; 153(15): 250-257.

[34] Xiao Q, Lim L T, Zhou Y, Zhao Z. Drying process of pullulan edible films forming solutions studied by low-field NMR. Food Chemistry, 2017; 230(1): 611-617.

[35] Li R, Li Z C, Chen S S, Yu J, Wang H Z, Zhang X L. Study of wter absorption of mung beans based on low-field nuclear magnetic resonance technology. Food Science, 2009; 30(15): 137-141.

[36] Li W, Wang P, Xu X, Xing T, Zhou G. Use of low-field nuclear magnetic resonance to characterize water properties in frozen chicken breasts thawed under high pressure. European Food Research \& Technology, 2014; 239(2): 183-188.

[37] Qi J, Gao F F, Li C B, Xu X L, Yang P Q. Changes of water holding capacity of mutton during freeze-thaw cycles by a low field nuclear magnetic resonance. Jiangsu Journal of Agricultural Sciences, 2010; 26(3): 617-622. (in Chinese)

[38] Yoder J, Malone M W, Espy M A, Sevanto S. Low-field nuclear magnetic resonance for the in vivo study of water content in trees. Review of Scientific Instruments, 2014; 85(9): 095110.

[39] Horn P J, Neogi P, Tombokan X, Ghosh S, Campbell B T, Chapman K D. Simultaneous quantification of oil and protein in cottonseed by low-field time-domain nuclear magnetic resonance. Journal of the American Oil Chemists' Society, 2011; 88(10): 1521-1529.

[40] Berman P, Leshem A, Etziony O, Levi O, Parmet Y, Saunders M, et al. Novel ${ }^{1} \mathrm{H}$ low field nuclear magnetic resonance applications for the field of biodiesel. Biotechnology for Biofuels, 2013; 6(1): 55.

[41] Sandnes R, Simon, Sébastien, Sjblom J, Srland F H. Optimization and validation of low field nuclear magnetic resonance sequences to determine low water contents and water profiles in W/O emulsions. Colloids and Surfaces A: Physicochemical and Engineering Aspects, 2014; 441(1): $441-448$. 